Check for updates

Cite this: Mater. Adv., 2020, 1,1330

Received 25th February 2020, Accepted 29th June 2020

DOI: 10.1039/d0ma00053a

rsc.li/materials-advances

\title{
Perylene diimide-based supramolecular polymer with temperature-sensitive ratiometric fluorescence responsiveness in solution and gels $\dagger$
}

\author{
Jie Liu, ${ }^{a}$ Yubing Zhang, ${ }^{a}$ Chonghua Zhang, ${ }^{a}$ Peisheng Zhang, (D) ${ }^{a}$ Rongjin Zeng, ${ }^{* a}$ \\ Jiaxi Cui (D) *b and Jian Chen (D)*ac
}

\begin{abstract}
A novel amphiphilic fluorescence building block with perylene diimide (PDI) as the core and quadruple $\mathrm{H}$-bonding groups (UPy) as wings (UPy-PDI-UPy) has been synthesized. It shows interesting thermoresponsive ratiometric dual-emission properties in both aqueous solution and the hydrogel state. Further contrast experiments with two other analogous derivatives, namely UPy-TPDI-UPy and TEG-PDI-TEG, indicated that the presence of supramolecular aggregation induced an emission enhancement effect between the UPy-PDI-UPy molecules, attributed to the synergetic effect of intermolecular intrinsic $\pi-\pi$ stacking, the hydrophobic effect and highly directional quadruple $\mathrm{H}$-bonding. In $\mathrm{DMF} / \mathrm{H}_{2} \mathrm{O}(\mathrm{v}: \mathrm{v}=1: 1)$, UPy-PDI-UPy self-assembles into nanoparticles with obvious ratiometric fluorescence responsiveness towards temperature in the range of $20-80^{\circ} \mathrm{C}$. Moreover, UPy-PDI-UPy can form a thermo-responsive hydrogel by dispersing in PEG-containing aqueous solution. The hydrogels show a temperaturedependent ratiometric dual-emission with a narrow responsive range of $20-39{ }^{\circ} \mathrm{C}$ and an excellent renewable property. This innovative research helps to fabricate novel responsive luminescent materials by using the supramolecular self-assembly behavior.
\end{abstract}

\section{Introduction}

Temperature-sensitive fluorescent materials are attracting increasing attention due to their application potential in fields from biosensing to information encryption..$^{1-4}$ In these applications, ratiometric fluorescence is preferred because it provides high resolution and allows accurate and quantitative readout in combination with a simple fluorescence change. ${ }^{5-15}$ Different material systems have been developed to get thermo-sensitive ratiometric fluorescence, including hybrid nanoparticles, carbon dots, polymer systems, rare-earth complexes, etc. ${ }^{10-15}$

\footnotetext{
${ }^{a}$ Key Laboratory of Theoretical Organic Chemistry and Functional Molecule of Ministry of Education, Hunan Provincial Key Laboratory of Controllable Preparation and Functional Application of Fine Polymers, Hunan Provincial Key Lab of Advanced Materials for New Energy Storage and Conversion, School of Chemistry and Chemical Engineering, Hunan University of Science and Technology, Xiangtan, Hunan, 411201, China. E-mail: zengrj1963@126.com, cj0066@gmail.com; Fax: +86731 58290045; Tel: +86 73158290045

${ }^{b}$ Institute of Fundamental and Frontier Sciences, University of Electronic Science and Technology of China, Chengdu, Sichuan, 610054, China. E-mail: jiaxicui119@gmail.com

${ }^{c}$ State Key Laboratory of Luminescent Materials and Devices, South China University of Technology, Guangzhou 510640, P. R. China

$\dagger$ Electronic supplementary information (ESI) available: Synthesis procedures, ${ }^{1} \mathrm{H}$ NMR spectra, ${ }^{13} \mathrm{C}$ NMR, MS, absorption spectrum, fluorescence spectra, etc. See DOI: $10.1039 /$ d0ma00053a
}

These systems either require complicated fabrication processes and extra calibration of physicochemical properties between the two different components or are unstable in polar solvents and show remarkable fluorescence quenching to oxygen. ${ }^{10-15}$ Fabricating ratiometric fluorescence thermo-sensing materials with single-component dyes could be a good solution to overcome these disadvantages. However, the design of such dyes is very challenging due to Kasha's rule.

The integration of dyes into a supramolecular system is an efficient method to create thermo-sensitive fluorescence materials. ${ }^{16-18}$ Among various supramolecular systems, supramolecular assemblies based on multiple $\mathrm{H}$-bonding interactions have been proven to be a promising way to construct novel fluorescent supramolecular polymers with controllable, stimuliresponsive, and reversible features. ${ }^{16-23}$ In these systems, the fluorophore moieties are conjugated with the H-bonding units to form fluorescence building blocks. ${ }^{16-23}$ When the temperature lowers to allow the formation of directional H-bonding to order the fluorophores, the materials normally show weak fluorescence due to the quenching effect. ${ }^{24}$ It is difficult to obtain ratiometric fluorescence, especially for the normal fluorophores that show obvious aggregation-caused quenching (ACQ) in the solid-state or high concentration of solution.

Perylene diimide (PDI) is one of the most well-known fluorescent building blocks to fabricate supramolecular 
fluorescence materials. ${ }^{25}$ It has been identified as an ideal candidate for fabricating organic optoelectronic devices, dye lasers, optical switches, and fluorescent sensors. ${ }^{25-31}$ And it exhibits numerous appealing features including easy functionalization, excellent stability, and ultrahigh fluorescence quantum yields in the molecular state. ${ }^{32-35}$ In spite of these advantages, traditional PDI-based supramolecular materials usually suffer from relatively weak fluorescent intensities of redshift emission, low quantum yields, and undesirable ACQ in aggregated states due to the strong intermolecular $\pi-\pi$ stacking of the PDI plane. ${ }^{36-39}$ Thus, the optimization of the supramolecular structure to modulate the $\pi-\pi$ stacking of PDI is essential to achieve bright emission in the aggregated state, which can be realized by the introduction of various aromatic rings as isolation groups from the sides. ${ }^{37,38}$ However, this strategy is often hindered by the complicated synthesis routes, and the obtained dyes generally exhibit a stationary red or deep red emission nature. ${ }^{40,41}$

Herein we report our strategy to prepare PDI-based ratiometric fluorescence supramolecular materials that are thermosensitive. In our strategy, the highly directional quadruple hydrogen bonding units of ureidopyrimidinone (UPy) are designed to conjugate with PDI to control the intermolecular $\pi-\pi$ stacking for endowing the dye with bright red emission in the aggregated state, together with a temperature-dependent ratiometric fluorescence property. We demonstrate this concept by comparing three rationally designed amphiphilic PDI derivatives, UPy-PDI-UPy, UPy-TPDI-UPy and TEG-PDI-TEG (Scheme 1), which are composed of a hydrophobic PDI core and two hydrophilic wings. Thanks to the unsubstituted core and linked quadruple H-bonding group (UPy) as wings, only the supramolecular polymer UPy-PDI-UPy exhibited an intense fluorescence emission in the solid-state and a temperaturedependent ratiometric dual-emission (green and red) property

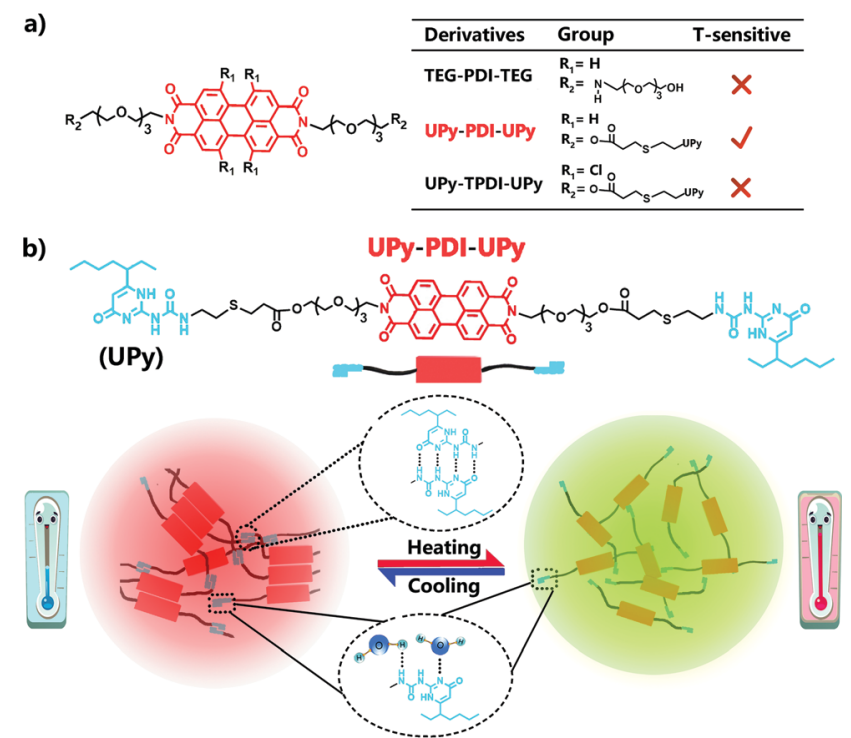

Scheme 1 (a) Molecular structure of three PDI derivatives; 2. (b) Schematic illustration of temperature-sensitive supramolecular fluorescent nanoassemblies. in the nanoparticle dispersion when temperature increased from 20 to $80{ }^{\circ} \mathrm{C}$. It is noteworthy that the subsequently prepared fluorescent hydrogel displayed a temperatureultrasensitive ratiometric fluorescence responsiveness and a visual sol-gel transition ranging from 20 to $39{ }^{\circ} \mathrm{C}$. To the best of our knowledge, this is the first example of the fabrication of PDI-based supramolecular polymers as ratiometric fluorescence thermometers and can imply more inspiration to prepare novel responsive luminescent materials (Scheme 1).

\section{Experimental}

\section{Materials and methods}

Unless otherwise stated, all the chemicals were purchased from commercial sources and directly used in the reaction. The detailed synthesis routes of relevant intermediates such as UPy-SH, OH-PDI-OH (2a) and OH-TPDI-OH (2b), as well as the final products including UPy-TPDI-UPy and TEG-PDI-TEG (Schemes S1-S3, ESI $\dagger$ ), and the relevant apparatus and characterization including NMR spectra, mass spectra (MS), UV-vis absorption and fluorescence spectra, fluorescence lifetime, atomic force microscopy (AFM) and control experiments are displayed in the ESI. $\dagger$

\section{Synthesis of $3 a$}

To a solution of 2a (742 mg, $1 \mathrm{mmol})$ in $500 \mathrm{~mL}$ dry $\mathrm{CH}_{2} \mathrm{Cl}_{2}$ (DCM, 4 A activated molecular sieves, 3 days) at $0{ }^{\circ} \mathrm{C}$, acryloyl chloride (109 mg, $1.2 \mathrm{mmol})$ and dry triethylamine $(\mathrm{NaOH}$, pellets 3 days) $(0.5 \mathrm{~mL}, 3.6 \mathrm{mmol})$ were added. The reaction mixture was stirred for $2 \mathrm{~h}$ at $0{ }^{\circ} \mathrm{C}$, and kept overnight at room temperature (RT) under an argon atmosphere. After the evaporation of the solvent, the product was purified by gel chromatography using $\mathrm{CH}_{2} \mathrm{Cl}_{2} / \mathrm{CH}_{3} \mathrm{OH}(50: 1$, v/v). 3a, a red solid, was obtained in $36 \%$ yield (286 mg). ${ }^{1} \mathrm{H}$ NMR (500 $\mathrm{MHz}, \mathrm{CDCl}_{3}$ ), $\delta: 8.61(\mathrm{~d}, 4 \mathrm{H}), 8.54(\mathrm{~d}, 4 \mathrm{H}), 6.38(\mathrm{~d}, 2 \mathrm{H}), 6.13(\mathrm{q}, 2 \mathrm{H}), 5.83(\mathrm{~d}, 2 \mathrm{H})$, $4.47(\mathrm{t}, 4 \mathrm{H}), 4.27(\mathrm{t}, 4 \mathrm{H}), 3.87(\mathrm{t}, 4 \mathrm{H}), 3.70-3.61(\mathrm{~m}, 20 \mathrm{H})$.

\section{Synthesis of UPy-PDI-UPy}

A mixture of 3a (152 mg, $0.18 \mathrm{mmol}), \mathrm{UPy}-\mathrm{SH}(170 \mathrm{mg}$, $0.54 \mathrm{mmol})$ and triethylamine $(0.2 \mathrm{~mL} 1.44 \mathrm{mmol})$ in $\mathrm{CH}_{2} \mathrm{Cl}_{2}$ $(30 \mathrm{~mL})$ was stirred under $\mathrm{N}_{2}$ at room temperature for $24 \mathrm{~h}$. After the evaporation of the solvent, the product was purified by gel chromatography using $\mathrm{CH}_{2} \mathrm{Cl}_{2} / \mathrm{CH}_{3} \mathrm{OH}(100: 1$, v/v) as the eluent to afford UPy-PDI-UPy $(120 \mathrm{mg})$. Yield: $45 \% .{ }^{1} \mathrm{H}$ NMR (500 MHz, $\left.\mathrm{CDCl}_{3}\right), \delta:$ 13.06-12.87 (m, 2H), 11.79-11.26 (m, 2H), 10.33-10.07 (m, 2H), 8.53-8.17 (m, 8H), 5.76-5.62 (m, 2H), 4.44 $(\mathrm{t}, 4 \mathrm{H}), 4.23(\mathrm{t}, 4 \mathrm{H}), 3.86(\mathrm{t}, 4 \mathrm{H}), 3.68-3.62(\mathrm{~m}, 20 \mathrm{H}), 3.40-3.24$ $(\mathrm{m}, 4 \mathrm{H}), 2.82-2.59(\mathrm{~m}, 12 \mathrm{H}), 2.28(\mathrm{~m}, 2 \mathrm{H}), 1.61-1.25(\mathrm{~m}, 16 \mathrm{H})$, 0.89-0.82 (m, 12H). MS (ESI): m/z 1745.6 [M] ${ }^{+}$(Fig. S1-S3, ESI $\dagger$ ).

\section{Results and discussion}

\section{Synthesis and characterization of PDI derivatives}

The chemical structures of the designed PDI derivatives are shown in Scheme 1. TEG-PDI-TEG consists of a PDI core and 
two hydrophilic 2-[2-[2-(2-aminoethoxy)ethoxy]ethoxy]ethanol (TEG) wings. UPy-PDI-UPy is made from a TEG-PDI-TEG structure and two ureido-4-pyrimidinone (UPy) terminal groups. Such a structure allowed UPy-PDI-UPy to form supramolecular polymers via the self-complementary quadruple H-bonding between the UPy moieties. ${ }^{18,19}$ Compared to UPy-PDI-UPy, UPy-TPDI-UPy has a tetrachloro-substituted PDI core. The substituted groups in TPDI were designed to weaken the $\pi-\pi$ stacking interaction of the PDI core. ${ }^{42,43}$ These PDI derivatives were synthesized by coupling TEG/UPy with bis- $N, N^{\prime}-(2-(2-(2-(2-$ hydroxyethoxy)ethoxy)ethoxy)ethyl)-3,4,9,10-perylenetetra-carboxylic diimide (OH-PDI-OH) or bis- $N, N^{\prime}$-(2-(2-(2-(2-hydroxyethoxy)ethoxy)ethoxy)ethyl)-1,6,7,12-tetrachlorol-3,4,9,10-perylenetetracarboxylic diimide (OH-TPDI-OH). The detailed synthesis pathways are described in the supporting information. The molecular structure of the three compounds and their intermediate products was confirmed by NMR spectra and mass spectra (Fig. S1-S8, ESI $\dagger$ ). All the three target molecules obtained exhibit good solubility in some organic solvents including dimethylformamide (DMF), dichloromethane (DCM), tetrahydrofuran (THF), etc., and in $\mathrm{DMF} / \mathrm{H}_{2} \mathrm{O}$ mixed solvent (in either molecular dispersion or aggregation state), due to the presence of the long hydrophilic chains.

Fig. 1 shows the fluorescent properties of the three PDI derivatives in the solid-state. Interestingly, under $365 \mathrm{~nm}$ light irradiation, UPy-PDI-UPy emits a bright red light with a high fluorescence quantum yield of $7.19 \%$ while both PEG-PDI-PEG and UPy-TPDI-UPy show weak fluorescence with low fluorescence quantum yields of $0.19 \%$ and $0.26 \%$, respectively (Fig. 1a). Such a unique property is supported by their fluorescence spectra (Fig. 1b) in which the maximum fluorescence emission intensity of UPy-PDI-UPy in the solid state at $694 \mathrm{~nm}$ is 53 times or 41 times higher than those of TEG-PDI-TEG at $693 \mathrm{~nm}$ or UPy-TPDI-UPy at $636 \mathrm{~nm}$. The absence of emission in both PEG-PDI-PEG and UPy-TPDI-UPy was attributed to the "aggregation-caused quenching" (ACQ) effect. It is well documented that the fluorescence of PDIs is generally quenched in the solid-state due to consecutive $\pi-\pi$ interactions and/or dipole-dipole interactions between the neighboring planar PDI fluorophores. ${ }^{39}$ Würthner and co-workers have demonstrated that bulky substituents on the PDI fluorophore favor the formation of discrete PDI-PDI $\pi$-dimers and undistorted planar PDI fluorophores, which inhibit long-range PDI
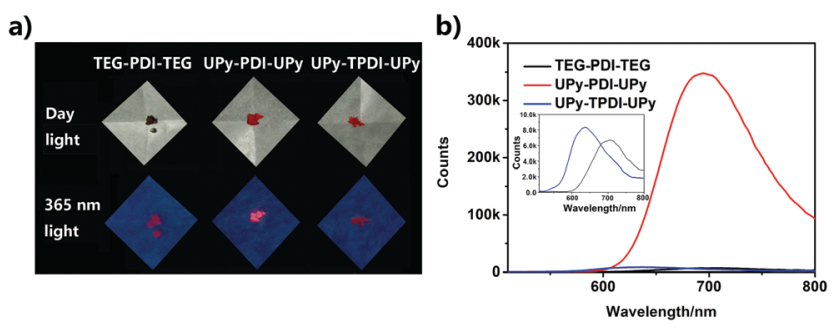

Fig. 1 (a) Photographs of TEG-PDI-TEG, UPy-PDI-UPy and UPy-TPDIUPy powder under daylight and $365 \mathrm{~nm}$ UV irradiation. (b) The corresponding fluorescence emission spectra of three molecules in the solid-state. $\lambda_{\mathrm{ex}}=490 \mathrm{~nm}$. aggregation in the solid state. ${ }^{44,45}$ As displayed in the ${ }^{1} \mathrm{H}$ NMR spectrum in Fig. S9 (ESI $\dagger$ ), with increasing the concentration of UPy-PDI-UPy, the peaks at 8.2 and $8.4 \mathrm{ppm}$ that belong to the PDI-PDI $\pi$-dimers gradually rise, indicating that the bulky UPy side groups in UPy-PDI-UPy can lead to the formation of discrete PDI-PDI $\pi$-dimers. ${ }^{46,47}$ Also, no chemical shifts related to trimers or other long-range PDI aggregation can be observed in Fig. S9 (ESI $\dagger$ ). We thus attribute the bright deep-red emission efficiency of UPy-PDI-UPy in the solid state to the appropriate $\pi-\pi$ stacking of PDI induced by UPy dimerization.

Because of their amphiphilic structures, the PDI derivatives could self-assemble in aqueous solutions. UV-vis absorption and fluorescence spectroscopies were used to study their selfassemblies (Fig. 2). A DMF/ $\mathrm{H}_{2} \mathrm{O}$ mixture solvent was employed by increasing the fraction of water in DMF. At a concentration of $50 \mu \mathrm{M}$, UPy-PDI-UPy completely dissolved in pure DMF, showing typical absorption and emission images of welldispersed PDI derivatives (Fig. 2a and b). ${ }^{48,49}$ When the content of water was increased to $30 \mathrm{vol} \%$, dramatic changes in both the absorption and emission spectra were observed. The $S_{0-1}$ transition $(\lambda=490 \mathrm{~nm})$ is more intense than the $S_{0-0}$ transition $(\lambda=525 \mathrm{~nm})$ while the fluorescent intensity at around $545 \mathrm{~nm}$ is significantly reduced, indicating a $\pi-\pi$ stacking aggregation state. With further increase of the water fraction, a slight bathochromic shift of $\lambda_{\max }$ occurred and the well-structured absorption curve turned into a less structured peak. The absorbance ratio of $A_{525} / A_{490}$ was declined from 1.6 to 0.4 (Fig. S10a, ESI $\dagger$ ), indicating enhanced $\pi-\pi$ stacking interaction. ${ }^{48}$ Interestingly, with
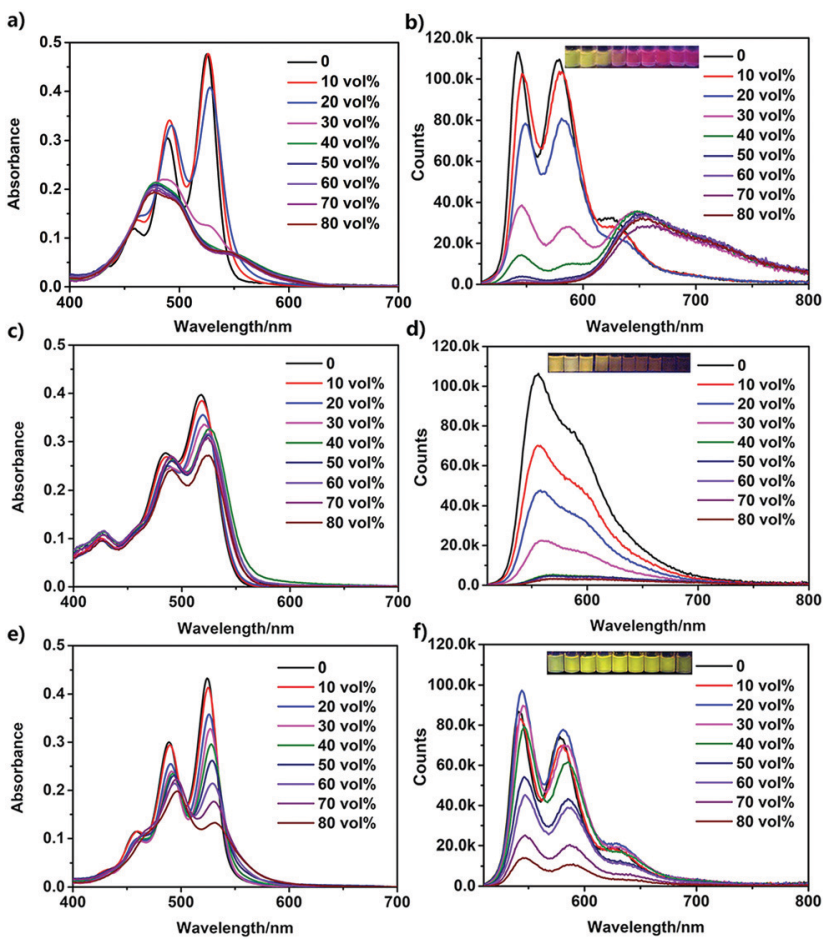

Fig. 2 UV-vis and fluorescence emission spectra of UPy-PDI-UPy (a and b), UPy-TPDI-UPy (c and d) and TEG-PDI-PDI-TEG (e and f) in different water fractions of $D M F / \mathrm{H}_{2} \mathrm{O}$ mixed solution $(50 \mu \mathrm{M})$, respectively. Fluorescence emission spectra: $\lambda_{\mathrm{ex}}=490 \mathrm{~nm}$. 
a further increase of water fraction, UPy-PDI-UPy shows a significant enhancement of fluorescence intensity at $646 \mathrm{~nm}$, accompanied by a change of emission color from green to red. This unusual enhancement in emission is different from most previous reports of the PDI derivatives, even UPy-linked ones. ${ }^{48,49}$ We attributed this to the formation of appropriate intermolecular $\pi-\pi$ stacking through multiple H-bonding interactions. Such a synergetic effect was supported by the results from UPy-TPDI-UPy in which the tetrachloro substituents prevent the intermolecular $\pi-\pi$ stacking interaction and TEG-PDI-TEG, a PDI derivative without UPy moieties. UPy-TPDI-UPy only exhibits a slight change in absorption along with the absorbance ratio of $A_{518} / A_{490}$ (Fig. S10b, ESI $\dagger$ ), and obvious fluorescence quenching at a higher water fraction (Fig. $2 \mathrm{c}$ and $3 \mathrm{~d}$ ). On the other hand, TEG-PDI-TEG shows an obvious decrease in the absorption peak at $\mathrm{S}_{0-0}$ transition (Fig. 2e) but a stepwise promotion in that of the $S_{0-1}$ transition. As a result, the absorbance ratio of $A_{525} / A_{490}$ declines from 1.45 to 0.7 (Fig. S10c, ESI $\dagger$ ). In addition to this change, clear quenching in fluorescence intensity was observed, due to the formation of strong $\pi-\pi$ interaction mediated aggregates, as reported in the literature.

To further investigate the aggregation behavior of UPy-PDIUPy in $\mathrm{DMF} / \mathrm{H}_{2} \mathrm{O}$ mixed solution, AFM, TEM and DLS were employed to study the size of its aggregatesunder different water fractions. As exhibited in Fig. 3 and Fig. S11 (ESI $\dagger$ ), when the water fraction was increased from $20 \%$ to $80 \%$, the particle size data from DLS gradually decrease and the size distribution was also reduced. The results were also confirmed by AFM and TEM measurements. Fig. 3a and c display the AFM images of the supramolecular aggregates of UPy-PDI-UPy in DMF/ $\mathrm{H}_{2} \mathrm{O}$ mixed solution with $30 \%$ or $50 \%$ fraction. In the case of $30 \%$ water fraction, the aggregates show an average size of $396.5 \mathrm{~nm}$ (DLS). Increasing water fraction reduces both the average particle size and size distribution. The TEM images as shown

a)
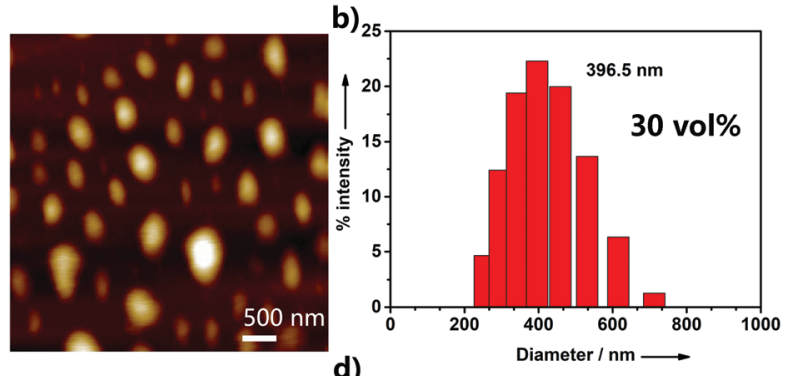

c)
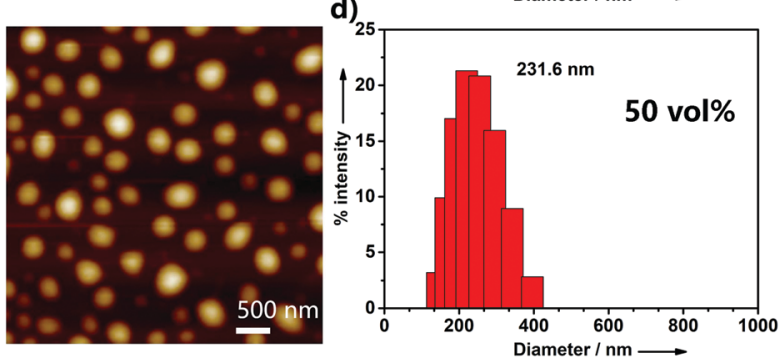

Fig. 3 AFM images and DLS distribution graph of the nanoparticles via the self-assembly of UPy-PDI-UPy $(50 \mu \mathrm{M})$ in $\mathrm{DMF} / \mathrm{H}_{2} \mathrm{O}$ mixed solution with a water fraction of 30 vol\% ( $a$ and $b$ ) and 50 vol\% (c and d), respectively. in Fig. S12 (ESI $\dagger$ ) further confirm the aggregation behavior of UPy-PDI-UPy in the mixture of DMF/ $\mathrm{H}_{2} \mathrm{O}$. This indicated that solvent polarity can be used to regulate the supramolecular selfassembly behavior of UPy-PDI-UPy in the current system.

\section{Ratiometric fluorescence responsiveness of temperature in solution and gels}

Temperature-dependent fluorescence emission spectra of the UPy-PDI-UPy nanoparticle dispersion in $\mathrm{DMF} / \mathrm{H}_{2} \mathrm{O}$ mixed solution $(\mathrm{v} / \mathrm{v}=50 / 50)$ were recorded from $20{ }^{\circ} \mathrm{C}$ to $80{ }^{\circ} \mathrm{C}$ (Fig. 4). With an increase in temperature, the fluorescence peak at $646 \mathrm{~nm}$ decreases and the emission around $545 \mathrm{~nm}$ becomes dominant (Fig. 4a). The average lifetime of the dispersion at $646 \mathrm{~nm}$ also declines from $15.9 \mathrm{~ns}$ to $8.9 \mathrm{~ns}$ (Fig. S13, ESI $\dagger$ ). The change in emission was attributed to the disassembly of PDI cores associated with both $\pi-\pi$ stacking and H-bonding interactions. The absorption spectra of the dispersion as shown in Fig. S14 (ESI $\dagger$ ) supported the conclusion that the $\pi-\pi$ stacking interaction was reduced with temperature promotion. In this process, particle size was reduced (Fig. S15, ESI $\dagger$ ), due to the segmental disaggregation of the formed supramolecular aggregates. Notably, a prominent linear fitting curve, with a correlation coefficient of $R^{2}=0.98$, was also achieved by plotting the logarithm of $I_{545} / I_{646}$ versus temperature $\left(20.0-80.0{ }^{\circ} \mathrm{C}\right)$. This perfect fitting implied that the nanoparticle dispersion can serve as a favorable ratiometric fluorescent sensor for temperature. In addition, when the temperature was alternated between 20 and $80{ }^{\circ} \mathrm{C}$, the nanoparticle dispersion exhibited reversibly switchable fluorescence nature (Fig. 4c). A slight "fatigue" effect was observed in reversibility (Fig. 4c) and fluorescence brightness (Fig. 5d). As shown in Fig. S16 (ESI $\dagger$ ), when the a)
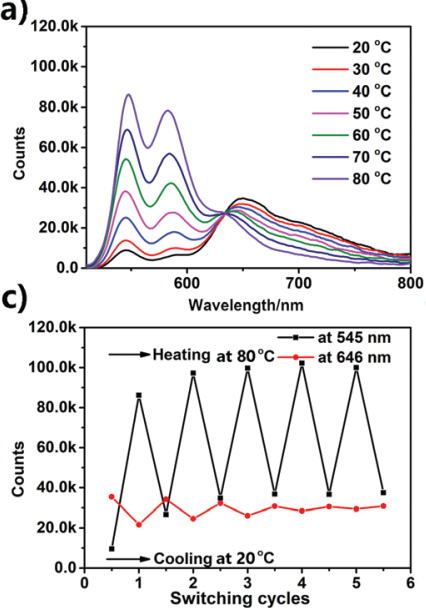

b)

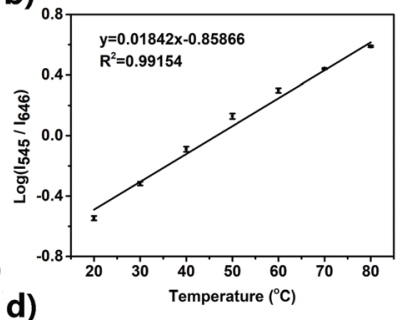

d)

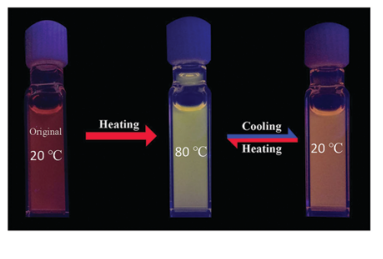

Fig. 4 (a) Temperature dependent fluorescence emission of the UPy-PDI-UPy $(50 \mu \mathrm{M})$ nanoparticle dispersion in $\mathrm{DMF} / \mathrm{H}_{2} \mathrm{O}: 50 / 50(\mathrm{v} / \mathrm{v})$. $\lambda_{\mathrm{ex}}=490 \mathrm{~nm}$. (b) The ratiometric fluorescence intensity $\left(/_{545} / /_{646}\right)$ of the nanoparticle dispersion versus temperature, as well as the corresponding linear fitting curve. (c) Renewable cycles of the corresponding fluorescence intensity at $546 \mathrm{~nm}$ and $649 \mathrm{~nm}$ change with cooling at $20^{\circ} \mathrm{C}$ and heating at $80{ }^{\circ} \mathrm{C}$. (d) Fluorescence photographs of the nanoparticle dispersion at the first cooling at $20{ }^{\circ} \mathrm{C}$ and the switchable fluorescence states between $20{ }^{\circ} \mathrm{C}$ and $80{ }^{\circ} \mathrm{C}$. 


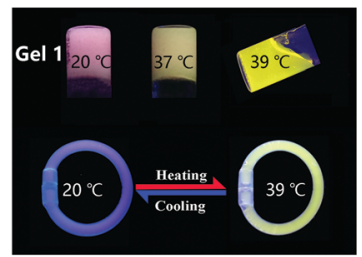

c)

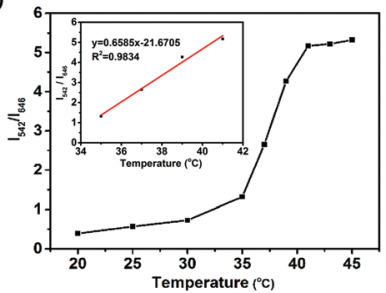

b)
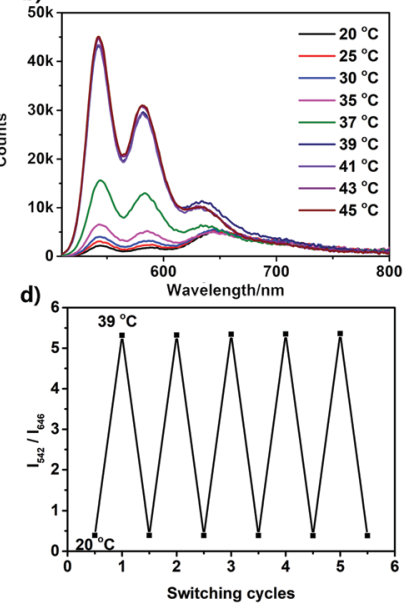

Fig. 5 (a) Fluorescence photographs of Gel1 at various temperatures, and the temperature-sensitive bracelet made of Gel1. (b) Temperaturedependent fluorescence emission of Gel1. $\lambda_{\text {ex }}=490 \mathrm{~nm}$. (c) The ratiometric fluorescence intensity $\left(/_{542} / /_{646}\right)$ of Gel1 versus temperature, and the corresponding linear fitting curve (inset). (d) Switching cycles of the $I_{542} / I_{646}$ of Gel1 between $20{ }^{\circ} \mathrm{C}$ and $39{ }^{\circ} \mathrm{C}$.

temperature was decreased from $80{ }^{\circ} \mathrm{C}$ to $20{ }^{\circ} \mathrm{C}$, some big UPyPDI-UPy nanoparticles form, which increases the distribution of nanoparticles. This result is significantly different from that of the nanoparticles formed in the mixture of $\mathrm{DMF} / \mathrm{H}_{2} \mathrm{O}$ solvent (Fig. 3c and d). The different assembly strategies make the color and the fluorescence emission spectra of the nanoparticles (Fig. 4d and Fig. S17, ESI $\dagger$ ) different from that of the initial sample at $20{ }^{\circ} \mathrm{C}$. In addition, the "fatigue" emission still exists, even when the incubation time of cooling was prolonged to 46 h (Fig. S17, ESI $\dagger$ ). However, marked fluorescent switching can still be visualized after five test cycles. In addition, the photostability of UPy-PDI-UPy nanoparticles in the mixture of $\mathrm{DMF} / \mathrm{H}_{2} \mathrm{O}$ is provided in Fig. S18 (ESI $\dagger$ ). The nanoparticles displayed good photostability under the continuous excitation of $490 \mathrm{~nm}$ for $60 \mathrm{~min}$. Thus, the nanoparticle dispersion exhibited attractive properties for renewable temperature sensing in various applications.

A UPy-PDI-UPy hydrogel could be prepared by adding polyethylene glycol (PEG, $\left.M_{\mathrm{W}}=10000\right)$ into the solution. This hydrogel (Gel1) was also thermo-sensitive. Elevating the temperature from 20 to $39{ }^{\circ} \mathrm{C}$ induced a sol-gel transition (Fig. 5a). Interestingly, Gel1 displays an obvious fluorescence color change from pink to greenyellow, accompanied by the sol-gel transition. The emission was further studied using fluorescence spectroscopy. As displayed in Fig. 5b, UPy-PDI-UPy in a hydrogel (Gel1) exhibits a high thermo-sensitivity in fluorescence, even more sensitive than that in the solution state. The ratiometric fluorescent intensity $I_{542} / I_{646}$ was further used to evaluate the thermo-sensitivity (Fig. 5c). It was found that a sharp change in $I_{542} / I_{646}$ (1.1 to 5.3) occurred in the range of $35-41{ }^{\circ} \mathrm{C}$ (critical temperature range). We attribute this to the phase-transition temperature of PEG in the as-prepared hydrogel intensifying the disaggregation of the formed supramolecular aggregates during the phase-transition process. Moreover, a good linear fitting curve with a correlation coefficient of

$0.98\left(R^{2}\right)$ was achieved by plotting the $I_{542} / I_{646}$ versus temperature (35.0-41.0 ${ }^{\circ} \mathrm{C}$ ). Note that this change perfectly matched the human physiological temperature range, implying a potential application in body-monitor devices. A temperature-sensitive color-changing bracelet by the encapsulation of Gel1 in a plastic tube was thus fabricated to show this potential (Fig. 5a). In addition, Gel1 also exhibits excellent reversibility in thermoinduced fluorescence change between 20 and $39{ }^{\circ} \mathrm{C}$ (Fig. 5d). In comparison with the corresponding nanoparticle dispersion, no detectable "fatigue" effects were observed in the reversibility even after five cycles. Furthermore, the critical temperature range of the gel can be easily varied by changing the ratio and molecular weight of PEG, as exhibited in Table S1 and Fig. S19-S21 (ESI $\dagger$ ). In the current system, PEG is the major component (58 and $75 \mathrm{wt} \%$ for Gel1 and Gel2, respectively) and water is absorbed in the PEG as a plasticizer in the gel state. PEG with $M_{\mathrm{w}}$ of 10000 shows a melting point of $c a .60{ }^{\circ} \mathrm{C}$, which is expected to decrease after the addition of water. The gel-sol transition is the melting of the mixture of PEG and water. This hypothesis is also supported by the system of the PEG with lower $M_{\mathrm{w}}$ (2000). Decreasing the $M_{\mathrm{w}}$ can reduce the melting point of PEG itself and also decrease the transition temperature of the system (system of $M_{\mathrm{w}} 10000: 41{ }^{\circ} \mathrm{C}$; system of $M_{\mathrm{w}}$ 2000: $36{ }^{\circ} \mathrm{C}$ ). Based on this mechanism, the concentration of PEG should make an important contribution to the phase transition, as the results show in Table S1, Fig. 5a and Fig. S19 (ESI $\dagger)\left(39{ }^{\circ} \mathrm{C}\right.$ for Gel1 and $41{ }^{\circ} \mathrm{C}$ for Gel2). In this system, the UPy-PDI-UPy molecules disperse in the media and would thus change their aggregated state with the media. Moreover, similar to UPy-PDI-UPy, TEG-PDI-TEG can also form a hydrogel. However, in contrast with the obvious temperaturedependence of UPy-PDI-UPy hydrogel, the corresponding TEGPDI-TEG in gel (Gel4, Table S1, Fig. S19 and S22, ESI $\dagger$ ) did not show any fluorescence color change except for the presence of fluorescence quenching and gel-sol transition with the increase of temperature.

\section{Conclusions}

In summary, we have synthesized three amphiphilic PDI derivatives, PEG-PDI-PEG, UPy-PDI-UPy, and UPy-TPDI-UPy. They have a PDI core and PEG/PEG-UPy wings. It was found that UPy-PDI-UPy exhibited an intense deep-red fluorescence emission not only in the solid-state, but also in the nanoparticle dispersion, implying an aggregation-induced-enhancement in fluorescence. We attributed such enhancement in fluorescence to the formation of appropriate $\pi-\pi$ stacking. Interestingly, UPy-PDI-UPy in a DMF/ $\mathrm{H}_{2} \mathrm{O}$ mixture solution (the volume ratio of $\mathrm{H}_{2} \mathrm{O}$ is $50 \%$ ) displayed obviously ratiometric fluorescence responsiveness against temperature in the range of $20-80{ }^{\circ} \mathrm{C}$, together with a good reversible cycling property. Moreover, the as-prepared fluorescent gels consisting of UPy-PDI-UPy and PEG not only exhibited visual sol-gel transition, but also showed temperature-sensitive ratiometric dual-emission signals at a narrow range of $20-39{ }^{\circ} \mathrm{C}$ with excellent renewable nature, which perfectly matched the human physiological temperature range. 
Those temperature-dependent ratiometric emission properties suggested potential application in various visual luminescent devices such as anti-counterfeiting labels and ratiometric fluorescent thermometers.

\section{Conflicts of interest}

The authors declare no competing financial interest.

\section{Acknowledgements}

We gratefully acknowledge the financial support by NSFC (51773056, 51873058, 51973023 and 51603067), Hunan Provincial Natural Science Foundation of China (2018JJ3143, 2020JJ3021), Key R \& D Program of Hunan Province (2019GK2237), China Postdoctoral Science Foundation (2017M622571 and 2018T110824), Open Project Program of State Key Laboratory of Chemo/Biosensing and Chemometrics (2016019) and the Open Fund of the State Key Laboratory of Luminescent Materials and Devices (South China University of Technology, 2019-skllmd-09), Bundesministerium für Bildung und Forschung under award number 031A360D.

\section{Notes and references}

1 A. Julià López, D. Ruiz-Molina, K. Landfester, M. B. Bannwarth and C. Roscini, Adv. Funct. Mater., 2018, 28, 1801492.

2 D. H. Liu, Z. B. Sun, Z. H. Zhao, Q. Peng and C. H. Zhao, Chem. - Eur. J., 2019, 25, 10179-10187.

3 J. Wang, N. Wang, G. Wu, S. Wang and X. Li, Angew. Chem., Int. Ed., 2019, 58, 3082-3086.

4 Z. Wang, X. He, T. Yong, Y. Miao, C. Zhang and B. Z. Tang, J. Am. Chem. Soc., 2020, 142, 512-519.

5 W. Zhang, X. Ji, B. J. Peng, S. Che, F. Ge, W. Liu, M. Al-Hashimi, C. Wang and L. Fang, Adv. Funct. Mater., 2019, 30, 1906463.

6 P. Zhang, H. Wang, Y. Hong, M. Yu, R. Zeng, Y. Long and J. Chen, Biosens. Bioelectron., 2018, 99, 318-324.

7 X. Peng, Z. Yang, J. Wang, J. Fan, Y. He, F. Song, B. Wang, S. Sun, J. Qu, J. Qi and M. Yang, J. Am. Chem. Soc., 2011, 133, 6626-6635.

8 H. Wang, P. Zhang, Y. Hong, B. Zhao, P. Yi and J. Chen, Polym. Chem., 2017, 8, 5795-5802.

9 E. Navarro Ceron, D. H. Ortgies, B. del Rosal, F. Ren, A. Benayas, F. Vetrone, D. Ma, F. Sanz-Rodriguez, J. Garcia Sole, D. Jaque and E. Martin Rodriguez, Adv. Mater., 2015, 27, 4781-4787.

10 J. Chen, C. Zhang, K. Lv, H. Wang, P. Zhang, P. Yi and J. Jiang, Sens. Actuators, B, 2017, 251, 533-541.

11 C. Wang, H. Lin, Z. Xu, Y. Huang, M. G. Humphrey and C. Zhang, ACS Appl. Mater. Interfaces, 2016, 8, 6621-6628.

12 D. Wang, T. Liu, J. Yin and S. Liu, Macromolecules, 2011, 44, 2282-2290.

13 Y. Takei, S. Arai, A. Murata, M. Takabayashi, K. Oyama, S. I. Ishiwata, S. Takeoka and M. Suzuki, ACS Nano, 2014, 8, 198-206.
14 M. Kamimura, T. Matsumoto, S. Suyari, M. Umezawa and K. Soga, J. Mater. Chem. B, 2017, 5, 1917-1925.

15 L. Li, Y. Zhu, X. Zhou, C. D. S. Brites, D. Ananias, Z. Lin, F. A. A. Paz, J. Rocha, W. Huang and L. D. Carlos, Adv. Funct. Mater., 2016, 26, 8677-8684.

16 A. Lavrenova, D. W. Balkenende, Y. Sagara, S. Schrettl, Y. C. Simon and C. Weder, J. Am. Chem. Soc., 2017, 139, 4302-4305.

17 S. Tomohiro, D. Arnab, T. Youichi and S. Seiji, J. Am. Chem. Soc., 2010, 132, 13928-13935.

18 H. Wang, W. Zhang, X. Dong and Y. Yang, Talanta, 2009, 77, 1864-1868.

19 H. Q. Peng, C. L. Sun, L. Y. Niu, Y. Z. Chen, L. Z. Wu, C. H. Tung and Q. Z. Yang, Adv. Funct. Mater., 2016, 26, 5483-5489.

20 Y. Y. Huang, Y. Tian, X. Q. Liu, Z. Niu, Q. Z. Yang, V. Ramamurthy, C. H. Tung, Y. Z. Chen and L. Z. Wu, Mater. Chem. Front., 2018, 2, 1893-1899.

21 J. F. Xu, Y. Z. Chen, D. Y. Wu, L. Z. Wu, C. H. Tung and Q. Z. Yang, Angew. Chem., Int. Ed., 2013, 52, 9738-9742.

22 X. Zhu, J. X. Wang, L. Y. Niu and Q. Z. Yang, Chem. Mater., 2019, 31, 3573-3581.

23 H. Q. Peng, J. F. Xu, Y. Z. Chen, L. Z. Wu, C. H. Tung and Q. Z. Yang, Chem. Commun., 2014, 50, 1334-1337.

24 G. Huang, Y. Jiang, S. Yang, B. S. Li and B. Z. Tang, Adv. Funct. Mater., 2019, 29, 1900516.

25 F. Wurthner, C. R. Saha-Moller, B. Fimmel, S. Ogi, P. Leowanawat and D. Schmidt, Chem. Rev., 2016, 116, 962-1052.

26 C. Li and H. Wonneberger, Adv. Mater., 2012, 24, 613-636.

27 W. Tan, X. Li, J. Zhang and H. Tian, Dyes Pigm., 2011, 89, 260-265.

28 S. Kaur, M. Kumar and V. Bhalla, Chem. Commun., 2015, 51, 4085-4088.

29 J. Yang, F. Chen, H. Ran, J. Y. Hu, B. Xiao, A. Tang, X. Wang and E. Zhou, Macromol. Rapid Commun., 2018, 39, 1700715.

30 L. Yang, W. Gu, L. Lv, Y. Chen, Y. Yang, P. Ye, J. Wu, L. Hong, A. Peng and H. Huang, Angew. Chem., Int. Ed., 2018, 57, 1096-1102.

31 Q. Zhao, K. Li, S. Chen, A. Qin, D. Ding, S. Zhang, Y. Liu, B. Liu, J. Z. Sun and B. Z. Tang, J. Mater. Chem., 2012, 22, 15128-15135.

32 D. Ke, A. Tang, C. Zhan and J. Yao, Chem. Commun., 2013, 49, 4914-4916.

33 A. Wadsworth, M. Moser, A. Marks, M. S. Little, N. Gasparini, C. J. Brabec, D. Baran and I. McCulloch, Chem. Soc. Rev., 2019, 48, 1596-1625.

34 W. Wagner, M. Wehner, V. Stepanenko and F. Würthner, J. Am. Chem. Soc., 2019, 141, 12044-12054.

35 E. Zhou, J. Cong, Q. Wei, K. Tajima, C. Yang and K. Hashimoto, Angew. Chem., Int. Ed., 2011, 50, 2799-2803.

36 P. M. H. Kazmaier and R. Hoffmann, J. Am. Chem. Soc., 1994, 116, 9684-9691.

37 S. K. Mohan Nalluri, J. Zhou, T. Cheng, Z. Liu, M. T. Nguyen, T. Chen, H. A. Patel, M. D. Krzyaniak, W. A. Goddard 3rd, M. R. Wasielewski and J. F. Stoddart, J. Am. Chem. Soc., 2019, 141, 1290-1303. 
38 Y. Yu, Q. Shi, Y. Li, T. Liu, L. Zhang, Z. Shuai and Y. Li, Chem. - Asian J., 2012, 7, 2904-2911.

39 H.-M. Zhao, J. Pfister, V. Settels, M. Renz, M. Kaupp, V. C. Dehm, F. Würthner, R. F. Fink and B. Engels, J. Am. Chem. Soc., 2009, 131, 15660-15668.

40 M. J. Lin, A. Jimenez, C. Burschka and F. Wurthner, Chem. Commun., 2012, 48, 12050-12052.

41 F. Schlosser, V. Stepanenko and F. Wurthner, Chem. Commun., 2010, 46, 8350-8352.

42 A. D. Shaller, W. Wang, H. Y. Gan and A. D. Q. Li, Angew. Chem., Int. Ed., 2008, 47, 7705-7709.

43 Á. J. Jiménez, M.-J. Lin, C. Burschka, J. Becker, V. Settels, B. Engels and F. Würthner, Chem. Sci., 2014, 5, 608-619.
44 M. M. Safont-Sempere, P. Osswald, K. Radacki and F. Würthner, Chem. - Eur. J., 2010, 16, 7380-7384.

45 M. M. Safont-Sempere, P. Osswald, M. Stolte, M. Grune, M. Renz, M. Kaupp, K. Radacki, H. Braunschweig and F. Würthner, J. Am. Chem. Soc., 2011, 133, 9580-9591.

46 W. Wang, L. S. Li, G. Helms, H. H. Zhou and A. D. Q. Li, J. Am. Chem. Soc., 2003, 125, 1120-1121.

47 W. Wang, W. Wan, H. H. Zhou, S. Q. Niu and A. D. Q. Li, J. Am. Chem. Soc., 2003, 125, 5248-5249.

48 L. L. Crowe, K. M. Solntsev and L. M. Tolbert, Langmuir, 2007, 23, 6227-6232.

49 W. Zhang, X. Zhou, Z. Xie, B. Yang, L. Liu and Y. Ma, Chem. Commun., 2013, 49, 11560-11562. 